\title{
Hyaluronic acid influence on platelet-induced airway smooth muscle cell proliferation
}

\author{
Ann-Charlotte Svensson Holm, Torbjörn Bengtsson, \\ Magnus Grenegård and Eva G Lindström
}

\section{Linköping University Post Print}

N.B.: When citing this work, cite the original article.

Original Publication:

Ann-Charlotte Svensson Holm, Torbjörn Bengtsson, Magnus Grenegård and Eva G Lindström, Hyaluronic acid influence on platelet-induced airway smooth muscle cell proliferation, 2012, Experimental Cell Research, (318), 5, 632-640.

http://dx.doi.org/10.1016/j.yexcr.2011.12.011

Copyright: Elsevier http://www.elsevier.com/

Postprint available at: Linköping University Electronic Press

http://urn.kb.se/resolve?urn=urn:nbn:se:liu:diva-75382 


\section{Hyaluronic acid influence on platelet-induced airway smooth muscle cell proliferation}

Ann-Charlotte B. Svensson Holm ${ }^{1,2}$, Torbjörn Bengtsson ${ }^{3}$, Magnus Grenegård ${ }^{1}$ \& Eva G. Lindström ${ }^{1}$

${ }^{1}$ Division of Drug Research/Pharmacology, Department of Medical and Health Sciences, Faculty of Health Sciences, Linköping University, SE-581 85 Linköping, Sweden

${ }^{2}$ Experimental Pathology, Department of Clinical and Experimental Medicine, Linköping University, SE-581 85 Linköping, Sweden

${ }^{3}$ Department of Biomedicine, School of Health and Medical Sciences, Örebro University, SE70182 Örebro, Sweden.

Running title: HA influence on platelet-induced ASMC proliferation

Correspondence and requests for reprints should be addressed to:

Ann-Charlotte Svensson Holm, Experimental Pathology, Department of Clinical and Experimental Medicine, Linköping University, SE-581 85 Linköping, Sweden Tel: +46-10-1031500

E-mail: ann-charlotte.svensson@liu.se. 


\section{Abstract}

Hyaluronic acid (HA) is one of the main components of the extracellular matrix (ECM) and is expressed throughout the body including the lung and mostly in areas surrounding proliferating and migrating cells. Furthermore, platelets have been implicated as important players in the airway remodeling process, e.g. due to their ability to induce airway smooth muscle cell (ASMC) proliferation. The aim of the present study was to investigate the role of HA, the HA-binding surface receptor CD44 and focal adhesion kinase (FAK) in plateletinduced ASMC proliferation. Proliferation of ASMC was measured using the MTS-assay, and we found that the CD44 blocking antibody and the HA synthase inhibitor 4Methylumbelliferone (4-MU) significantly inhibited platelet-induced ASMC proliferation. The interaction between ASMC and platelets was studied by fluorescent staining of F-actin. In addition, the ability of ASMC to synthesise HA was investigated by fluorescent staining using biotinylated HA-binding protein and a streptavidin conjugate. We observed that ASMC produced HA and that a CD44 blocking antibody and 4-MU significantly inhibited platelet binding to the area surrounding the ASMC. Furthermore, the FAK-inhibitor PF 573228 inhibited platelet-induced ASMC proliferation. Co-culture of ASMC and platelets also resulted in increased phosphorylation of FAK as detected by Western blot analysis. In addition, 4-MU significantly inhibited the increased FAK-phosphorylation. In conclusion, our findings demonstrate that ECM has the ability to influence platelet-induced ASMC proliferation. Specifically, we propose that HA produced by ASMC is recognised by platelet CD44. The platelet/HA interaction is followed by FAK activation and increased proliferation of co-cultured ASMC. We also suggest that the mitogenic effect of platelets represents a potential important and novel mechanism that may contribute to airway remodelling. 
Keywords: airway smooth muscle; airway remodeling, extracellular matrix, hyaluronic acid, CD44, focal adhesion kinase, platelets 


\section{Introduction}

Platelets have been suggested to play a part in the chronic inflammatory disease asthma where different structural changes, such as increased cell number (goblet cells, fibroblasts and airway smooth muscle cells (ASMC)), cause airway remodeling. Platelets have been shown to interact with different cell types, e.g. by recruiting leucocytes through P-selectin in a murine model of chronic allergic inflammation $[1,2]$. We have recently found that platelets have the ability to bind to ASMC and induce a significant proliferation [3]. We have also observed that different platelet preparations, e.g. platelet membranes, significantly stimulate ASMC proliferation [4]. These results suggest that not only soluble factors but that the interaction between platelets and ASMC are of importance for the mitogenic effect of platelets. However, the mechanisms involved are far from understood.

Hyaluronic acid (HA), a glycosaminoglycan, is one of the main components of the extracellular matrix (ECM). HA is composed of repeating units of GlcNAc- $\beta(1-4)-G l c U A-$ $\beta(1-3)$ and produced by e.g. smooth muscle cells by three different HA synthases (HAS 1-3) using UDP-GlcUA and UDP-GlcNac as substrates [5, 6]. HA is expressed throughout the body including the lung mostly in areas surrounding proliferating and migrating cells and especially during inflammation and tissue repair [7]. HA is therefore believed to play an important role in both proliferation and migration of cells $[8,9]$. HA has recently been shown to bind to different receptors, e.g. CD44, a type 1 transmembrane receptor expressed on the surface of smooth muscle cells and platelets. CD44 exists in many different isoforms that all have HA binding properties [10] and regulate several signaling pathways including Src family kinases, Rho family GTPases, extracellular signal-regulated kinases and mitogen activated protein kinase (MAPK) [11, 12] and thereby mediates both cell adhesion and cell growth [13, 14]. 
CD44 has a structural role in linking the ECM to the cytoskeleton and thereby regulates cell shape and motility [15-17], i.e. properties resembling those of focal adhesions. Focal adhesion kinase (FAK) is a non-receptor protein tyrosine kinase expressed in most cell types, such as smooth muscle cells and platelets. FAK is recruited at an early stage to focal adhesions and mediates many downstream responses. FAK is an adaptor for protein-protein interactions, and transmit thereby adhesion and growth factor-dependent signals into the cell [18]. FAK has been shown to regulate many different signaling pathways by affecting phosphoinositide 3kinase, phospholipase C, Rho GTPases and MAPK [19, 20].

The aim of the present study was to investigate the importance of HA, CD44 and FAKdependent signaling in the interaction between platelets and ASMC and their role in plateletinduced cell proliferation. Increased knowledge regarding ECM and intracellular signaling pathways involved in the interaction between platelets and ASMC might in the future lead to potential important and novel mechanism that may contribute to airway remodelling. 


\section{Materials and methods}

\section{Chemicals}

The chemicals used were as follows: Dulbecco's Modified Eagle Medium, non-essential amino acids, sodium pyruvate, penicillin and streptomycin (PEST), foetal bovine serum (FBS) and Trypsin-EDTA (Gibco, Paisley, Scotland); CellTiter $96^{\circledR}$ Aqueous One solution cell proliferation assay (Promega, Madison, WI, USA); streptavidin Alexa Fluor ${ }^{\circledR} 488$ conjugate and Alexa Fluor ${ }^{\circledR}$ 594-phalloidin (Molecular Probes, Eugene, OR, USA); lysophosphatidylcholine (LPC), platelet-derived growth factor (PDGF) and 4methylumbelliferone (4-MU) (Sigma Chemical Co., St. Louis, MO, USA); CD44 blocking antibody, Protease Inhibitor Cocktail, EDTA-free (100X) and Halt ${ }^{\mathrm{TM}}$ Phosphatase Inhibitor Cocktail, 100X (Thermo Fisher Scientific, Fremont, CA, USA); Biotinylated HA binding protein (Merck, Darmstadt, Germany); Antiphosphotyrosine, clone 4G10 (Millipore, Billerica, MA, USA); goat anti-mouse IgG-HRP (Santa Cruz Biotechnology, Santa Cruz, CA, USA); Vectashield (Vector Laboratories Inc, Burlingame, CA, USA); purified mouse antiFAK (BD Transduction Laboratories ${ }^{\mathrm{TM}}$, San Jose, CA, USA); protein A Sepharose CL-4B (GE Healthcare, Little Chalfont, UK); paraformaldehyde (PFA) (Labkemi, Stockholm, Sweden); mouse IgG1 negative control (AbD Serotec, Oxford, UK); PF 573228 (Tocris, Bristol, UK).

\section{Buffers and media}

The following buffers and media were used in the experiments: phosphate-buffered saline $\mathrm{pH}$ 7.3 (PBS; $137 \mathrm{mM} \mathrm{NaCl}, 27 \mathrm{mM} \mathrm{KCl}, 6.74 \mathrm{mM} \mathrm{Na}_{2} \mathrm{HPO}_{4} \times 2 \mathrm{H}_{2} 0,1.47 \mathrm{mM} \mathrm{KH}_{2} \mathrm{PO}_{4}$ and 0.5\% BSA); PBS pH 7.3 (1.2 \% BSA); Krebs-Ringer phosphate buffer pH 7.3 (KRG; 120 $\mathrm{mM} \mathrm{NaCl}, 4.9 \mathrm{mM} \mathrm{KCl}, 1.2 \mathrm{mM} \mathrm{MgSO}_{4}, 1.7 \mathrm{mM} \mathrm{KH}_{2} \mathrm{PO}_{4}, 8.3 \mathrm{mM} \mathrm{Na}_{2} \mathrm{HPO}_{4}, 1 \mathrm{mM} \mathrm{CaCl}_{2}$ and $10 \mathrm{mM}$ glucose); KRG without $\mathrm{CaCl}_{2}$; acid citrate/dextrose solution (ACD; $85 \mathrm{mM}$ 
$\mathrm{C}_{6} \mathrm{H}_{5} \mathrm{Na}_{3} \mathrm{O}_{7}, 71 \mathrm{mM} \mathrm{H} \mathrm{H}_{3} \mathrm{C}_{6} \mathrm{H}_{5} \mathrm{O}_{7}$ and $111 \mathrm{mM}$ D-glucose); ); RIPA buffer (2\% Triton X-100, $2 \%$ sodium deoxoycholate, $0.2 \%$ SDS, $316 \mathrm{mM} \mathrm{NaCl}, 2 \mathrm{mM}$ EGTA, $20 \mathrm{mM}$ Tris-HCl); starvation medium (DMEM, 1mM sodium pyruvate, $1 \%$ non-essential amino acids, $100 \mathrm{U} / \mathrm{ml}$ penicillin, and $100 \mu \mathrm{g} / \mathrm{ml}$ streptomycin); complete medium (starvation medium with $10 \%$ foetal bovine serum).

\section{Cell culture}

Human ASMC (Promocell, Heidelberg, Germany) were cultured in complete medium in a humidified atmosphere at $37^{\circ} \mathrm{C}$ and $5 \% \mathrm{CO}_{2}$. ASMC displayed all the reported characteristics of viable smooth muscle cells in culture when examined by light microscopy [21]. ASMC in passages 9 to 20 were used.

\section{Platelet preparation}

Fresh blood from healthy donors was obtained from the blood bank at Linköping University Hospital, Linköping, Sweden and platelets were isolated as previously described [3]. In short, five parts of the blood were mixed with one part of ACD solution and centrifuged for 20 min at $220 \mathrm{x}$ g. The platelet-rich plasma obtained in the upper layer was removed and centrifuged for $20 \mathrm{~min}$ at $480 \mathrm{x}$ g. The platelet pellet was gently washed and resuspended in KRG without calcium, and the platelets were counted in a Bürker chamber.

\section{ASMC proliferation}

ASMC proliferation was measured as previously described [3]. Briefly ASMC (3000/well) were seeded in 96-well plates. After $24 \mathrm{~h}$ the medium was changed to starvation medium and incubated for $24 \mathrm{~h}$. Thereafter, cells were incubated for further $24 \mathrm{~h}$ in medium supplemented with $0.1 \%$ FBS, in the absence (controls) or presence of platelets (ASMC/platelet ratio of 
1/1000) and drugs $(0.1-10 \mu \mathrm{g} / \mathrm{ml}$ of the CD44 blocking antibody, $10 \mu \mathrm{g} / \mathrm{ml}$ of the mouse IgG1 negative control antibody, 100-600 $\mu \mathrm{M}$ of the HAS-inhibitor 4-Methylumbelliferone (4-MU) and $0.01-1 \mu \mathrm{M}$ of the FAK-inhibitor PF 573228). Inhibitors were added to both cell types 20 min prior co-incubation. ASMC density and ASMC/platelet ratio were based on previous studies $[3,4]$.

ASMC proliferation was analysed using the CellTiter96 ${ }^{\circledR}$ Aqueous One Solution Cell Proliferation Assay (MTS-assay). Briefly, after incubation with the stimulus, new medium supplemented with $0.1 \%$ FBS was added together with the CellTiter $96^{\circledR}$ Aqueous One Solution Reagent, and the amounts of viable cells were measured spectrophotometrically at $490 \mathrm{~nm}$ using a microplate reader (Spectra MAX 340, Molecular Devices, Sunnyvale, CA) [22]. All drug and solvents used were tested for interference with the assay.

\section{Synthesis of HA and microscopic examination of the ASMC/platelet interaction}

The ability of ASMC to synthesise HA was investigated by fluorescent staining using biotinylated HA-binding protein $(\mathrm{HBP})$ and Alexa Fluor ${ }^{\circledR} 488$ streptavidin conjugate. In addition, the interaction between ASMC and platelets was studied morphologically by fluorescent staining of the F-actin using Alexa Fluor ${ }^{\circledR}$ 594-phalloidin and the nucleus using DAPI conjugated Vectashield followed by fluorescence microscopy. ASMC (10 000/well) were seeded in an 8-well chamber slide (Nunc, Rochester, NY, USA) in complete medium and incubated for $24 \mathrm{~h}$ in a humidified atmosphere at $37^{\circ} \mathrm{C}$ and $5 \% \mathrm{CO}_{2}$ followed by $24 \mathrm{~h}$ of serum deprivation. Thereafter, cells were incubated for $2 \mathrm{~h}$ in DMEM supplemented with $0.1 \%$ FBS, in the absence (controls) or presence of platelets (ASMC/platelet ratio of 1/1000) and drugs $(10 \mu \mathrm{g} / \mathrm{ml} \mathrm{CD44} \mathrm{blocking} \mathrm{antibody,} \mathrm{100-600} \mu \mathrm{M}$ 4-MU and $1 \mu \mathrm{M}$ PF 573228). Inhibitors were added to both cell types 20 min prior co-incubation. After $2 \mathrm{~h}$ of coincubation 
the cells were treated with $4 \%$ paraformaldehyde for 30 min in RT followed by incubation with biotin-conjugated $\mathrm{HBP}(1: 100)$ for $45 \mathrm{~min}$ at $37^{\circ} \mathrm{C}$. Afterwards the cells were incubated with streptavidin-conjugated Alexa 488 (1:250) for $45 \mathrm{~min}$ at RT. Thereafter the samples were permeabilised and F-actin stained in a mixture of lysophosphatidylcholine $(100 \mu \mathrm{g} / \mathrm{ml})$ and Alexa Fluor ${ }^{\circledR} 594$-phalloidin $(8 \mu \mathrm{g} / \mathrm{ml})$ in PBS pH 7.3. The chamber slides were mounted with coverslips using DAPI conjugated Vectashield and placed in the refrigerator prior to fluorescence microscopy analysis (Carl Zeiss, Oberkochen, Germany).

Quantification of the amount HBP/image and F-actin/image was made using Image J v. 1.45 (available as freeware from http://rsbweb.nih.gov/ij/). The degree of platelet in close association to ASMC (no visible space between the ASMC and the platelets) and the degree of platelets bound to the area surrounding the ASMC was quantified by manual counting in the fluorescence microscope by an expert in the method. Platelets on total 6-9 images per treatment (3 independent experiments and 2-3 replicates/experiment) were counted and morphologically evaluated whether they were in close association to the ASMC or bound to the area surrounding the ASMC. All pictures had identical settings to enable equivalent image analysis.

\section{Western blot of FAK}

ASMC (100 000/well) were seeded in 6-well plates in complete medium and incubated for 24 $\mathrm{h}$ in a humidified atmosphere at $37^{\circ} \mathrm{C}$ and $5 \% \mathrm{CO}_{2}$ followed by $24 \mathrm{~h}$ of serum deprivation. Thereafter, cells were incubated for 1 hour in medium supplemented with $0.1 \%$ FBS, in the absence (controls) or presence of platelets (ASMC/platelet ratio of 1/1000) and drugs (10 $\mu \mathrm{g} / \mathrm{ml} \mathrm{CD44} \mathrm{blocking} \mathrm{antibody,} 600 \mu \mathrm{M} 4-\mathrm{MU}$ and 0.1 and $1 \mu \mathrm{M}$ PF 573228) or $150 \mathrm{ng} / \mathrm{ml}$ PDGF. Inhibitors were added to both cell types 20 min prior co-incubation. The cells (both 
ASMC and platelets) were thereafter lysed by incubation in RIPA buffer supplemented with 1X phosphatase and protease inhibitor coctail for $30 \mathrm{~min}$ on ice followed by centrifugation at $14000 \mathrm{~g}$ at $4^{\circ} \mathrm{C}$ for $10 \mathrm{~min}$. The supernatant was incubated with $0.5 \mu \mathrm{g}$ FAK antibody for $2 \mathrm{~h}$ at $4^{\circ} \mathrm{C}$ followed by immunoprecipitation over night at $4^{\circ} \mathrm{C}$ using protein $\mathrm{A}$ sepharose. The protein A sepharose/antibody mix was washed three times, diluted in sample buffer and thereafter heated to $95^{\circ} \mathrm{C}$ for $5 \mathrm{~min}$. The samples were separated on a NuPAGE ${ }^{\circledR} 3-8 \%$ TrisAcetate gel (Invitrogen, Carlsbad, CA, USA) using an Invitrogen Novex ${ }^{\circledR}$ Mini-cell. The proteins were transferred to a PVDF membrane using a Mini Trans-Blot Electrophoretic Transfer Cell (Bio-Rad, Hercules, CA, USA). The membranes were blocked for $1 \mathrm{~h}$ in RT with 5\% (w/v) dry milk and $0.1 \%$ Tween 20 in PBS pH 7.4 to minimise nonspecific binding. The membranes were thereafter incubated for $1 \mathrm{~h}$ at RT with a primary mouse antiphosphotyrosine 4G10 antibody (diluted 1:200 in PBS supplemented with 1\% (w/v) BSA, $0.1 \%(\mathrm{v} / \mathrm{v})$ Tween 20 and $0.02 \%(\mathrm{w} / \mathrm{v})$ sodium acid) and subsequently incubated with horseradish peroxidase-conjugated goat anti-mouse IgG secondary antibody (diluted 1:2000 in $0.1 \%$ (v/v) Tween 20 in PBS) for $1 \mathrm{~h}$. HRP antibody-conjugated proteins were visualised by chemiluminescence using Immobilon ${ }^{\mathrm{TM}}$ Western Chemiluminescence HRP substrate (Millipore, Billerica, MA, USA).

\section{Statistical analysis}

Results are normalised in relation to unstimulated ASMC and are expressed as mean values (\% of control) \pm standard error of the mean (S.E.M). One-way ANOVA followed by Dunnet's multiple comparison tests was used for statistical analysis. A p-value $<0.05$ was considered to be significant, as denoted $\left.{ }^{*}(\mathrm{p}<0.05)\right)^{* *}(\mathrm{p}<0.01)$ and ${ }^{* * *}(\mathrm{p}<0.001)$. Data were analysed using GraphPad Prism ${ }^{\mathrm{TM}}$ (GraphPad Software, San Diego, CA). 


\section{Results}

The role of HA in platelet-induced ASMC proliferation

We have recently observed that platelets have the capacity to bind to ASMC [3] and that platelet-induced ASMC proliferation in our experimental setup is mainly mediated by membrane bound structures [4] suggesting that a physical interaction between platelets and ASMC is important for the mitogenic effect of platelets. ASMC were therefore stimulated with platelets at an ASMC/platelet ratio of 1/1000 in the presence or absence of a CD44 blocking antibody $(0.1-10 \mu \mathrm{g} / \mathrm{ml})$ and the HAS-inhibitor 4-MU (100-600 $\mu \mathrm{M})$. Proliferation was measured using the MTS-assay and the ASMC/platelet ratio was chosen based on previous findings [3].

The results indicate that the presence of platelets causes a significant increase in the proliferation of ASMC after $24 \mathrm{~h}$ of coincubation (Figure 1 and 2A). Interestingly, our data demonstrated that platelet-induced ASMC proliferation was significantly reduced by $0.1-10$ $\mu \mathrm{g} / \mathrm{ml}$ of a CD44 blocking antibody (Figure 1) and by $600 \mu \mathrm{M}$ of 4-MU (Figure 2A). Importantly, none of the drugs (the blocking CD44 antibody; Figure 1 inset, 4-MU; Figure 2A inset) affected basal ASMC proliferation significantly and $10 \mu \mathrm{g} / \mathrm{ml}$ of an IgG1 isotype antibody did not affect platelet-induced ASMC proliferation $(151.9 \pm 0.72, \mathrm{n}=3)$.

The ability of ASMC to synthesise HA was thereafter investigated by staining HA using biotinylated HA-binding protein and Alexa Fluor ${ }^{\circledR} 488$ streptavidin conjugate followed by fluorescence microscopy. We found that ASMC synthesise considerable amounts of HA (Figure 2B I, 3 independent experiments and 2-3 replicates/experiment). Incubation with 600 $\mu \mathrm{M}$ of the HAS inhibitor 4-MU significantly inhibited HA production in ASMC (Figure 2B II, 2C). Control experiments conducted to investigate the specificity of biotin-conjugated HA- 
Figure 1
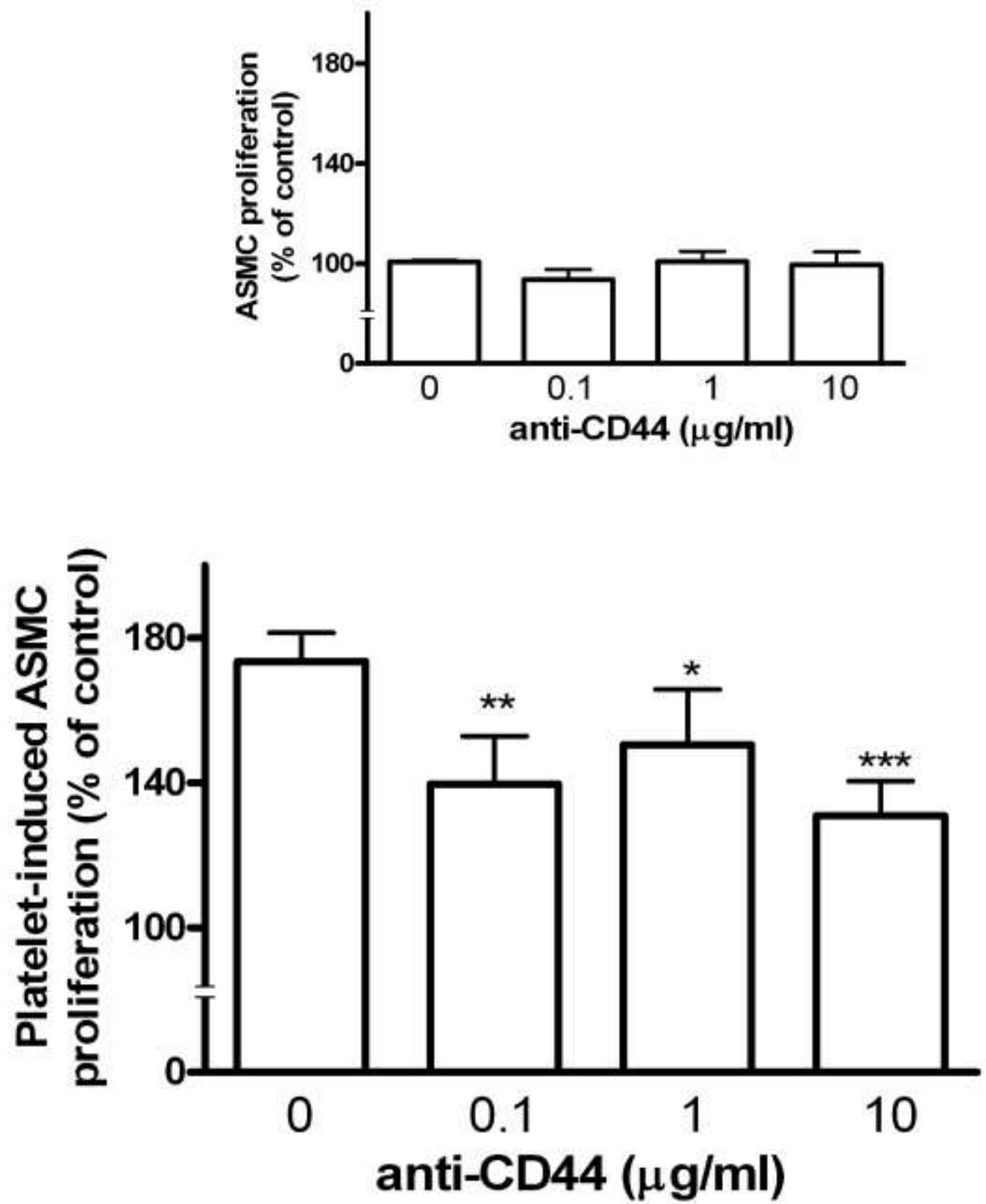

Figure 1. Effect of a CD44 blocking antibody on platelet-induced ASMC proliferation. The platelet-mediated increase in ASMC proliferation, measured using the MTS-assay, was significantly inhibited by the CD44 blocking antibody $(n=4-7)$. The inset shows that the CD44 blocking antibody did not have an effect on basal ASMC proliferation. Data are expressed as means \pm SEM and One-way ANOVA followed by Dunnet's multiple comparison tests was used for statistical analysis in this and for the data in the following figures. 
Figure 2A
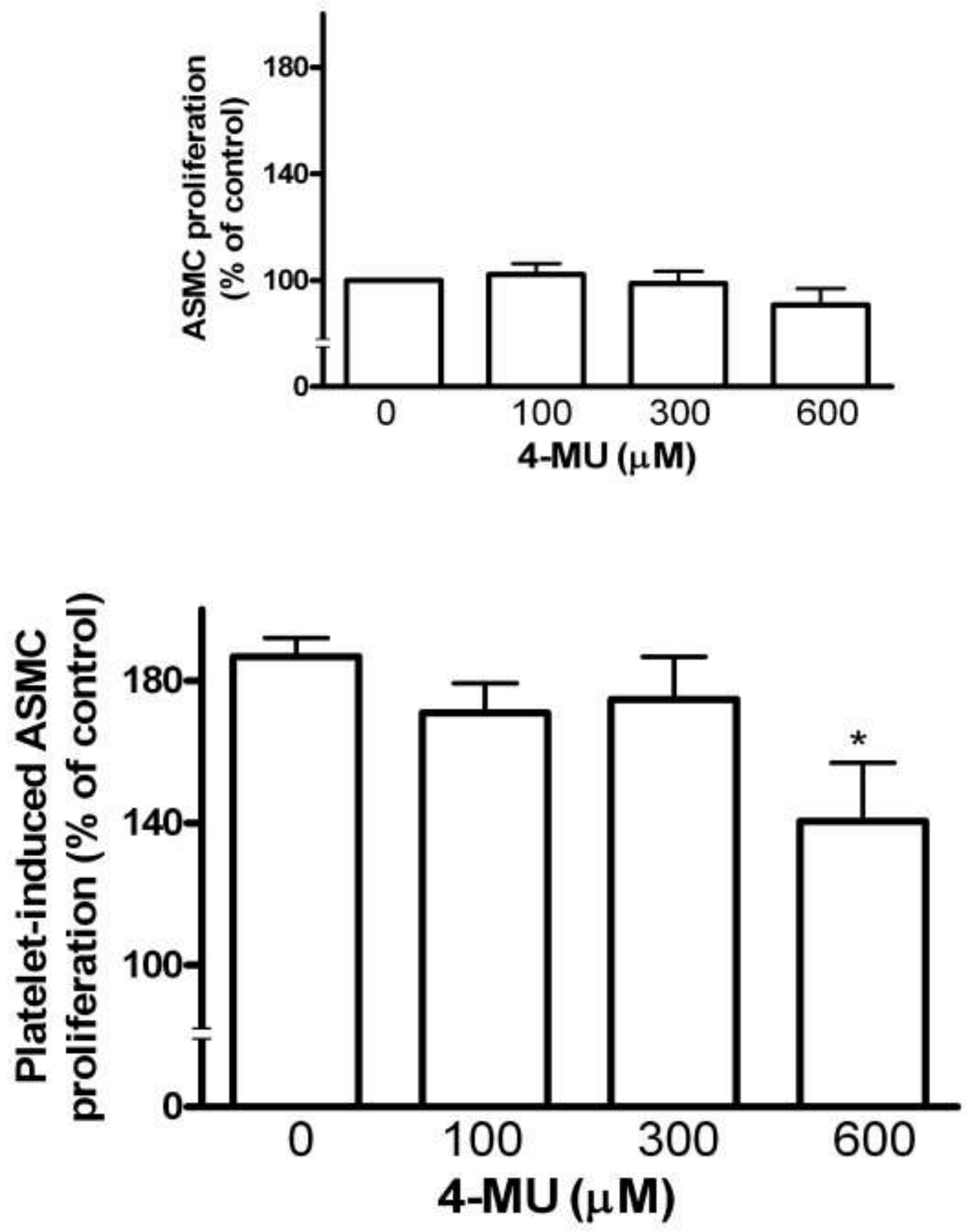
Figure 2B

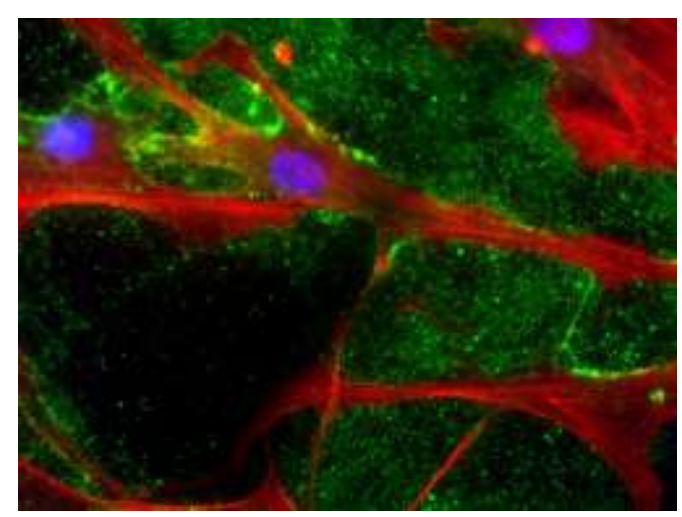

I: ASMC

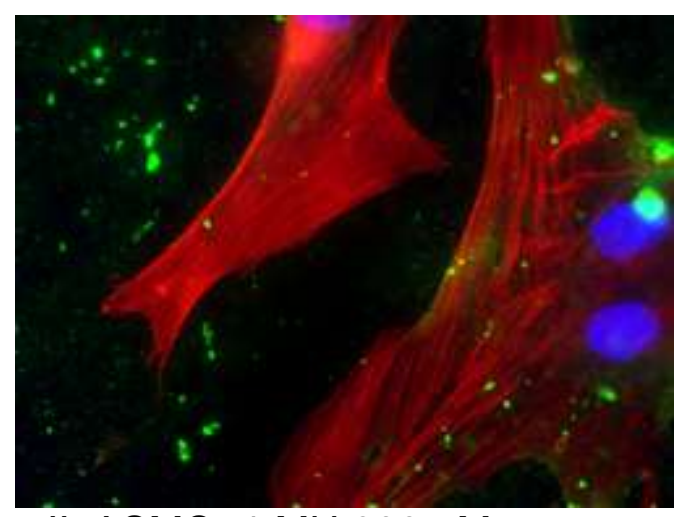

II: ASMC+4-MU $600 \mu \mathrm{M}$

C

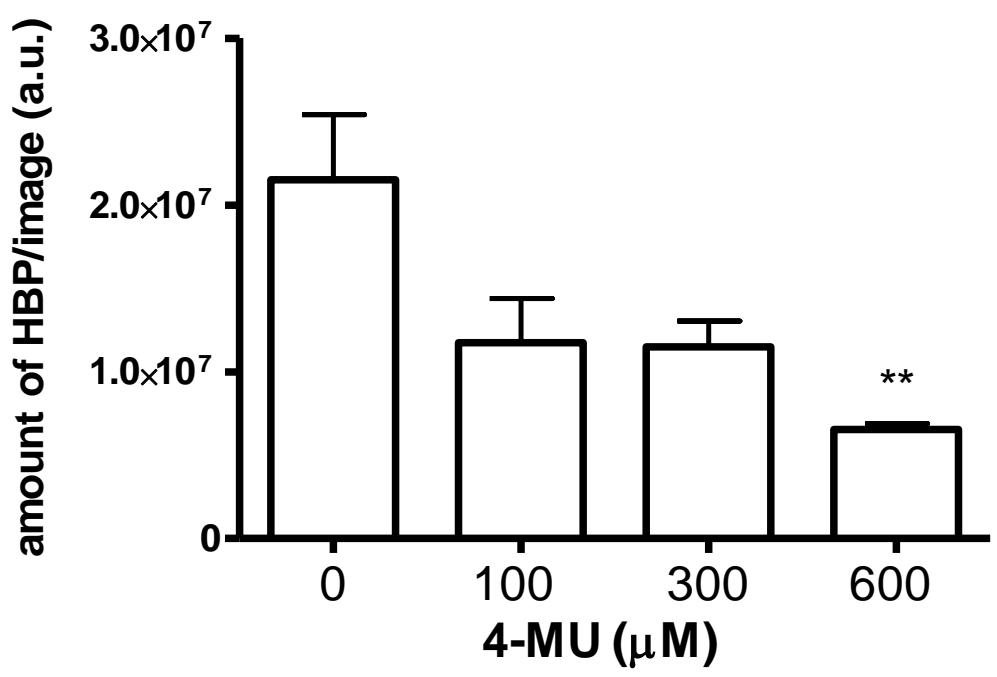

Figure 2. A: Effect of 4-MU on platelet-induced ASMC proliferation. The platelet-mediated increase in ASMC proliferation, measured using the MTS-assay, was significantly inhibited by $600 \mu \mathrm{M} 4-\mathrm{MU}(\mathrm{n}=5-6)$. The inset shows that 4-MU did not affect basal ASMC proliferation. B: Synthesis of HA. ASMC produce HA (I). In addition, 4-MU inhibits HA produced by ASMC after $2 \mathrm{~h}$ of incubation. (II) HA (green), F-actin (red) and the nucleus (blue) is fluorescent stained as described in the Materials and methods section. The images shown are representatives of 3 independent experiments (2-3 replicates/experiment) obtained from different cell passages. C: Quantification of the amount HBP/image using Image J. 600 $\mu \mathrm{M}$ 4-MU significantly inhibits HA synthesis in ASMC. 
binding protein and streptavidin-conjugated Alexa 488 revealed no nonspecific binding and ASMC morphology and F-aktin levels was not affected by the drug treatments (data not shown).

The role of $H A$ and $C D 44$ in the interaction between platelets and ASMC

As previously presented, we observed that platelets induce a CD44 and HA-dependent ASMC proliferation and that ASMC produce HA. Consequently, we wanted to investigated the role of HA and CD44 in the interaction between platelets and ASMC. This was done by incubating ASMC for $2 \mathrm{~h}$ in the absence (controls) or presence of platelets and $10 \mu \mathrm{g} / \mathrm{ml}$ CD44 blocking antibody or $600 \mu \mathrm{M}$ 4-MU followed by fluorescent staining of HA, the actin cytoskeleton and the nucleus of the ASMC (Figure 3, 3 independent experiments and 3 replicates/experiment). We found that platelets bound to both ASMC and the HA-rich area surrounding the ASMC (Figure 3A II). In addition, $10 \mu \mathrm{g} / \mathrm{ml}$ of the CD44 blocking antibody and $600 \mu \mathrm{M}$ 4-MU significantly inhibited the ability of platelets to bind to the area surrounding ASMC (Figure 3A III-IV and 3B II).

\section{The role of FAK in platelet-induced ASMC proliferation}

FAK regulates many different signaling pathways by affecting e.g. G-protein linked receptors and transmembrane receptors for different growth factors [23]. The role of FAK in plateletinduced ASMC proliferation was investigated using the FAK inhibitor PF 573228. We found that platelet-induced ASMC proliferation was significantly inhibited by 0.1 and $1 \mu \mathrm{M}$ of PF 573228 (Figure 4A). $1 \mu \mathrm{M}$ of PF 573228 also significantly reduced basal ASMC proliferation (Figure 4A, inset). PF 573228 did not affect the morphology of ASMC or the binding between platelets and ASMC (data not shown). 
Figure 3A

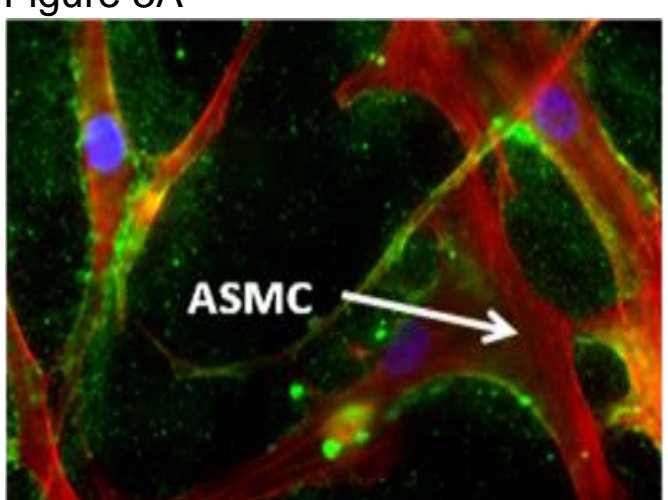

I: ASMC

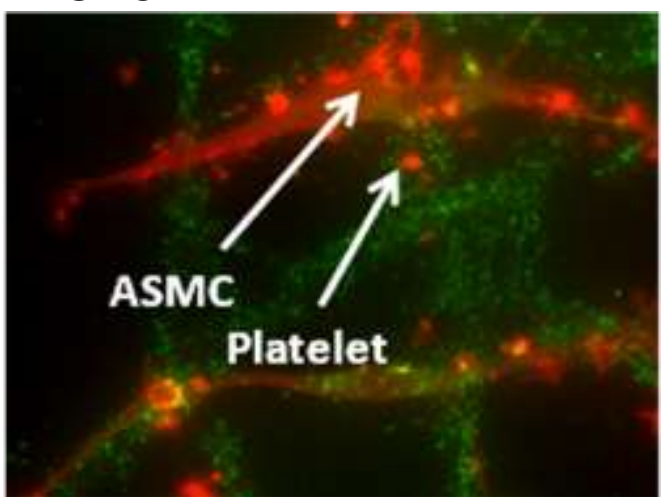

III: ASMC+platelets+anti CD44 $10 \mu \mathrm{g} / \mathrm{ml}$ $\mathrm{B}$

I: Degree of platelets in close
proximity to ASMC proximity to

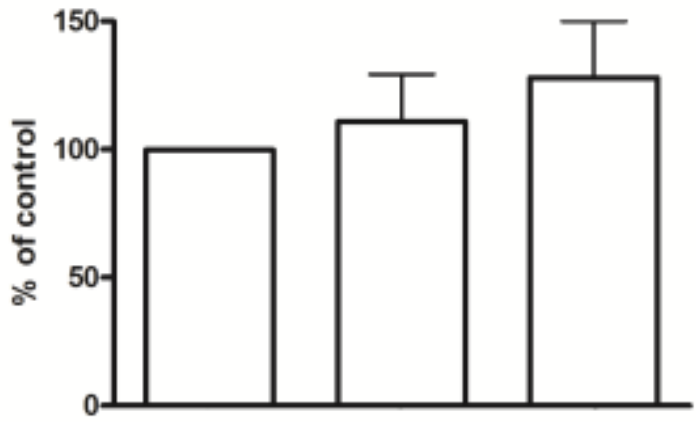

ASMC

Platelets

anti-CD44 $10 \mu \mathrm{g} / \mathrm{ml}$

4-MU $600 \mu \mathrm{M}$

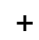

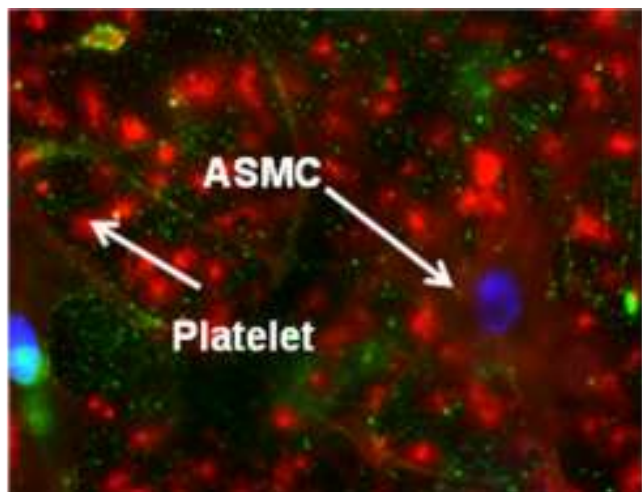

\section{II: ASMC+platelets}

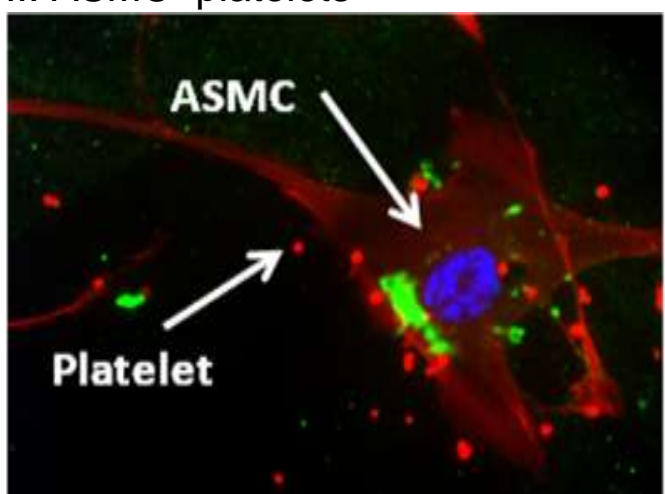

IV: ASMC+platelets+4-MU $600 \mu \mathrm{M}$

II: Degree of platelets bound to the area surrounding the ASMC

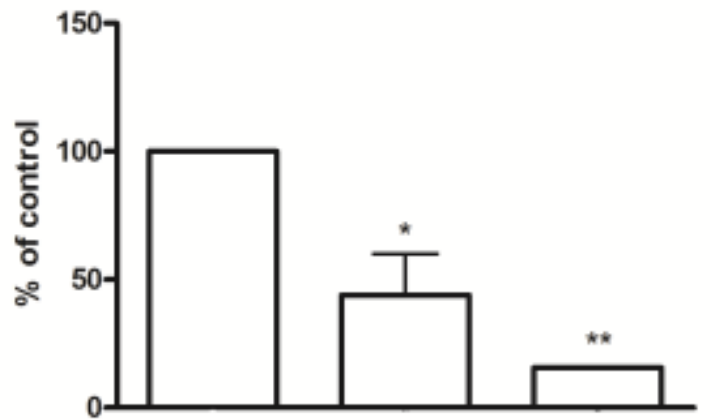

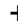

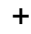

Figure 3. Effects of 4-MU and a CD44 blocking antibody on ASMC/platelet interaction.

ASMC were incubated in the presence or absence of platelets and 4-MU (600 $\mu \mathrm{M})$ or anti-CD44 (10 $\mu \mathrm{g} / \mathrm{ml})$. A I: ASMC. A II: Platelets bind to both ASMC and the HA-rich area surrounding the ASMC. In addition, $10 \mu \mathrm{g} / \mathrm{ml}$ of the blocking CD44 antibody and $600 \mu \mathrm{M} 4$-MU significantly inhibited the ability of platelets to bind to the area surrounding ASMC (A III-IV and B II). HA (green), F-actin (red) and the nucleus (blue) is fluorescent stained as described in the Materials and methods section. The images shown are representatives of 3 independent experiments (2-3 replicates/experiment) obtained from different cell passages and blood donors. White arrows indicate platelets and ASMC. Quantification of platelets in close proximity of ASMC and platelets bound to the area surrounding the ASMC was made by manual counting and is expressed as percent of control (ASMC+platelets). 
Figure 4
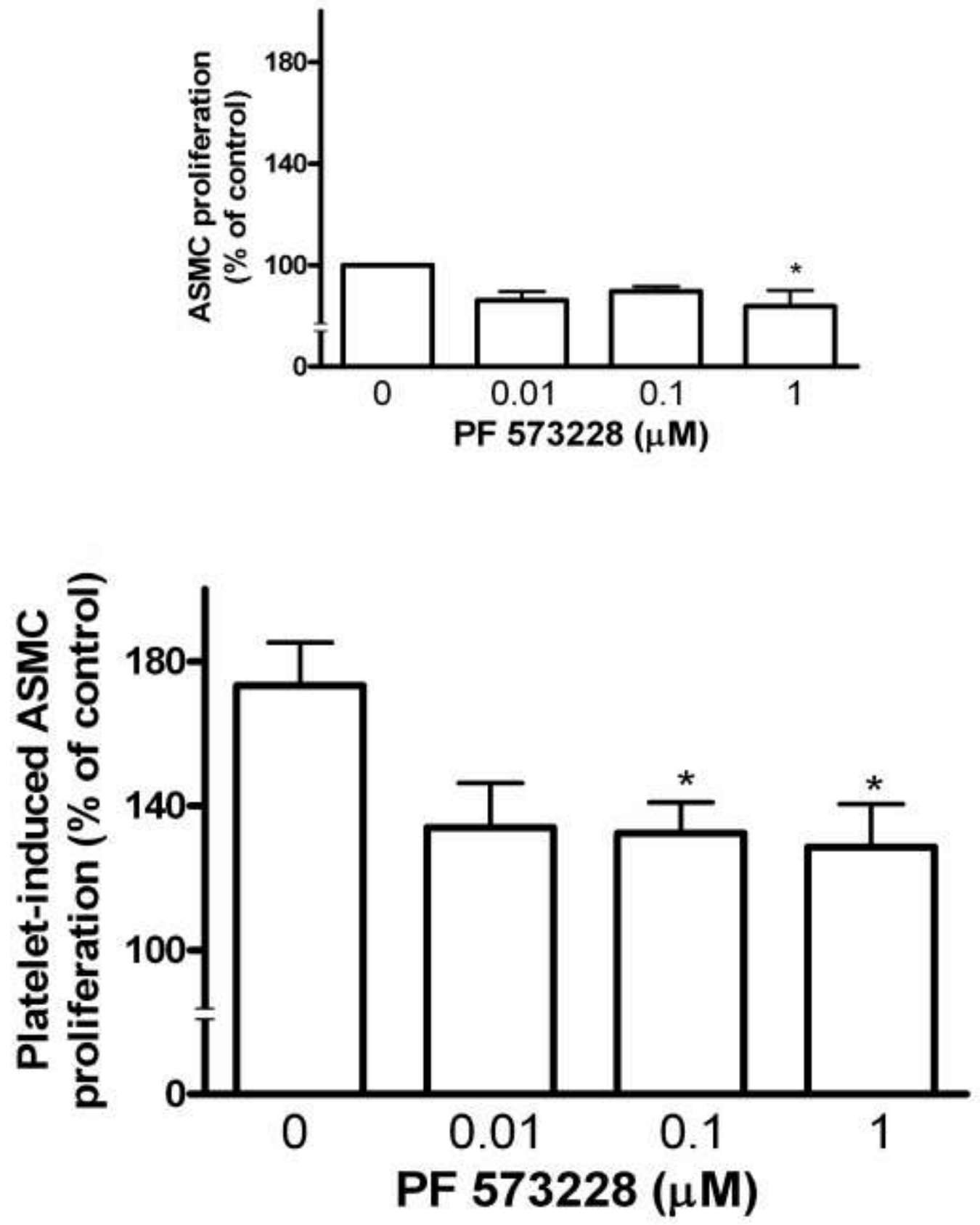

Figure 4. Effect of PF 573228 on platelet-induced ASMC proliferation. Platelet-induced ASMC proliferation was significantly inhibited by 0.1 and $1 \mu \mathrm{M}$ of PF $573228(\mathrm{~A}, \mathrm{n}=3-5)$, measured using the MTS-assay. The inset shows that $1 \mu \mathrm{M}$ PF 573228 also reduced basal ASMC proliferation. 
Phosphorylation of immunoprecipitated FAK after $1 \mathrm{~h}$ of coincubation between platelets and ASMC (ASMC/platelet ratio of 1/1000) in the presence or absence of 0.1 and $1 \mu \mathrm{M}$ PF 53228 was detected using Western blot analyses. We found that phosphorylated FAK was significantly increased upon coincubation of ASMC with platelets $(195.5 \pm 27.4, n=4, p<$ 0.05). We also found a significant reduction of FAK phosphorylation (generated after coincubation between platelets and ASMC) when ASMC and platelets were treated with 1 $\mu \mathrm{M}$ of the FAK inhibitor in PF $573228(119.5 \pm 15.4, \mathrm{n}=3, \mathrm{p}<0.05)$. PDGF $(150 \mathrm{ng} / \mathrm{ml})$, used as a positive control, also induced FAK phosphorylation in ASMC, which was reduced by $1 \mu \mathrm{M}$ PF 573228 (data not shown).

It has previously been shown that FAK is phosphorylated and thereby activated as a consequence of interaction between ECM and surface receptors such as integrins [24]. We were therefore interested in studying whether the CD44 blocking antibody and the HASinhibitor 4-MU affected the platelet-induced FAK phosphorylation in ASMC. We found that $600 \mu \mathrm{M}$ 4-MU significantly inhibited FAK phosphorylation generated after $1 \mathrm{~h}$ of coincubation between platelets and ASMC, while the CD44 blocking antibody did not have any significant effect (Figure 5). 
Figure 5

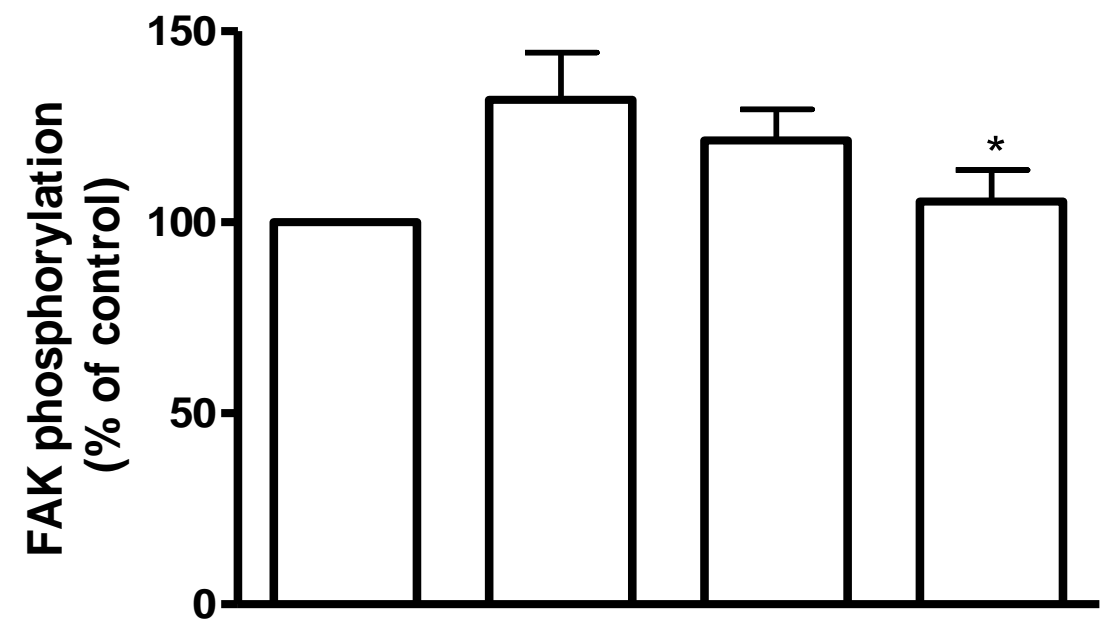

phospho-FAK

$\begin{array}{lllll}\text { ASMC } & + & + & + & + \\ \text { Platelets } & - & + & + & + \\ \text { anti CD44 10 } & - & - & + & - \\ \mu \mathrm{g} / \mathrm{ml} & & & & + \\ \text { 4-MU 600 } \mu \mathrm{M} & - & - & - & +\end{array}$

Figure 5. Effect of a CD44 blocking antibody and 4-MU on FAK phosphorylation. Western blot analyses showed that 4-MU significantly inhibited the FAK phosphorylation generated after coincubation of ASMC and platelets. Expression of phospho FAK was analysed using a primary mouse antiphosphotyrosine 4G10 antibody followed by a secondary horseradish peroxidase conjugated goat anti-mouse IgG antibody. Prior to Western blotting, the amount of DNA in each sample of ASMC was determined. The blot is representative of 4 independent experiments obtained from different cell passages and blood donors. Data obtained from the quantitative analyses of the phospho FAK bands were normalised to ASMC, set to $100 \%$ and are expressed as means \pm SEM. 


\section{Discussion}

Platelets contribute to airway inflammation and facilitate structural changes, such as increased cell proliferation, observed during the remodeling process $[25,26]$. We have previously found that platelets as well as platelet-derived membrane preparations stimulate ASMC proliferation measured using the MTS-assay, thymidine incorporation, measurement of DNA content and manual cell counting $[3,4]$. This implies that a close association between platelets and ASMC is essential for the increased proliferative activity. However, the role played by specific surface receptors and their corresponding ligands and intracellular signaling pathways are unknown. The present study elucidated the possible role of HA (one of the main ECM component), the HA-binding receptor CD44 and FAK in platelet-induced proliferation of ASMC.

It has previously been shown that platelets bind to HA via surface expressed CD44 [27]. In our study, it was found that a CD44 blocking antibody significantly inhibited the ability of platelets to bind to the HA-rich area surrounding the ASMC. Furthermore, the HAS-inhibitor 4-MU (which reduced HA-synthesis) also decreased the amount of platelets adhering to the surface between the ASMC. The HAS-inhibitor as well as the CD44 blocking antibody significantly inhibited the mitogenic effect of platelets in co-cultures with platelets and ASMC, without affecting ASMC proliferation in the absence of platelets. Interestingly, the drugs used did not inhibit direct interaction between platelets and ASMC. 
Taken together, we suggest that CD44 mediates platelet/HA binding, and that this represent one of the crucial events for the increased proliferation of co-cultured ASMC. Our results also indicate that part of the pro-proliferative effect of platelets is due to CD44-independent interactions with the ASMC. Furthermore, it has been shown that T-lymphocytes increase DNA synthesis in ASMC through a mechanism involving CD44 [28]. HA has a molecular mass ranging between 1-10 $000 \mathrm{kDa}$ and the high molecular weight HA (HMW-HA, 500$10000 \mathrm{kDa})$ is produced by HAS 1-3 while low molecular weight HA (1-500 kDa) is produced by enzymatic degradation of HMW-HA by hyaluronidases (HYAL) or through oxidative hydrolysis by reactive oxygen species (ROS) of HMW-HA [29, 30]. Platelets possess HYAL-2 that, in cooperation with CD44, may cleave HA into small pro-proliferative fragments [31-34]. Interestingly, we recently found that the platelet/ASMC interaction results in increased ROS-production [3]. Consequently, it is tempting to speculate that platelets facilitate degradation of HA into smaller pro-proliferative fragments. However, the exact link between platelet/HA interaction and the subsequent increase in ASMC proliferation remains to be determined.

Previous studies have indicated that the HA-binding receptor CD44 possess properties resembling those of focal adhesion molecules, e.g. participation in the regulation of cell morphology and motility [15-17]. Furthermore, it has been shown that FAK is phosphorylated and thereby activated as a consequence of interaction between ECM and surface receptors such as integrins, and thereby influence proliferation and cell migration [24]. In the present study, we found that the platelet-induced ASMC proliferation was significantly inhibited by the competitive FAK inhibitor PF 573228. In accordance, it has been reported that antisense oligonucleotides directed against FAK inhibited proliferation of pulmonary artery smooth muscle cells and glioma cells $[35,36]$. In the present study, morphological analyses revealed 
that PF 573228 had no effect on the interaction between platelets and ASMC. This suggests that PF 573228 suppress platelet-induced ASMC proliferation without reducing the binding of platelets to HA and ASMC.

We also found that the HAS inhibitor 4-MU, but not the blocking CD44 antibody, significantly inhibited FAK phosphorylation (detected using Western blot analyses) generated after coincubation between platelets and ASMC. This indicates that platelet binding to HA is an initial and important step that ultimately results in FAK-activation. It has previously been suggested that HA is able to induce FAK phosphorylation [20, 37], FAK-dependent activation of MAPK and secretion of the inflammatory mediator metalloproteinase-2 [20, 37]. Taken together, we propose that platelet/HA interaction is a prerequisite for subsequent FAKactivation in a co-culture of ASMC and platelets. Furthermore, our results demonstrated that FAK activation generated in a co-culture of ASMC and platelets is associated with increased proliferation of ASMC.

We have previously described that platelets binding to ASMC may be pivotal for the proproliferative effect of the former cell type $[3,4]$. In light of our novel findings it is possible that platelets bound to the HA-rich area surrounding the ASMC are the main reason for the observed mitogenic effect. However, it should be emphasised that ROS as well as LOX activity may contribute to ASMC proliferation in a co-culture of platelets and ASMC [3, 4]. From a broad perspective, it is therefore obvious that the mitogenic action of platelets is multifactorial. The precise connection between HA, CD44, ROS, FAK and LOX activities in the cross-talk between platelets and ASMC remains to be determined 
In conclusion, our findings demonstrated that ECM has the ability to influence plateletinduced ASMC proliferation. Specifically, we propose that HA produced by ASMC is recognised by platelet CD44. The platelet/HA interaction is followed by FAK activation and increased proliferation of co-cultured ASMC. We also suggest that the mitogenic effect of platelets is partly due to CD44-independent interaction with ASMC. This action of platelets represents a potential important and novel mechanism that may contribute to airway remodeling. The results may also have impact in the development of new pharmacological strategies in the treatment of patients with inflammatory airway disorders. 


\section{Acknowledgements}

This work was supported by grants from strategic areas Cardiovascular Inflammation

Research Centre (CIRC) and Material in Medicine, the Heart- and Lung foundation, the County of Östergötlands Län, STIFUD, Olle E and the Swedish Research Council.

\section{Disclosures}

The authors declare that they have no conflict of interest. 


\section{References}

[1] S.C. Pitchford, H. Yano, R. Lever, Y. Riffo-Vasquez, S. Ciferri, M.J. Rose, S. Giannini, S. Momi, D. Spina, B. O'Connor, P. Gresele, C.P. Page, Platelets are essential for leukocyte recruitment in allergic inflammation, J Allergy Clin Immunol 112 (2003) 109-118.

[2] S.C. Pitchford, S. Momi, S. Giannini, L. Casali, D. Spina, C.P. Page, P. Gresele, Platelet P-selectin is required for pulmonary eosinophil and lymphocyte recruitment in a murine model of allergic inflammation, Blood 105 (2005) 2074-2081.

[3] A.C. Svensson Holm, T. Bengtsson, M. Grenegard, E.G. Lindstrom, Platelets stimulate airway smooth muscle cell proliferation through mechanisms involving 5lipoxygenase and reactive oxygen species, Platelets 19 (2008) 528-536.

[4] A.C. Svensson Holm, T. Bengtsson, M. Grenegard, E.G. Lindstrom, Platelet membranes induce airway smooth muscle cell proliferation, Platelets 22 (2011) 43-53.

[5] A. Kultti, S. Pasonen-Seppanen, M. Jauhiainen, K.J. Rilla, R. Karna, E. Pyoria, R.H. Tammi, M.I. Tammi, 4-Methylumbelliferone inhibits hyaluronan synthesis by depletion of cellular UDP-glucuronic acid and downregulation of hyaluronan synthase 2 and 3, Exp Cell Res 315 (2009) 1914-1923.

[6] A. Aruffo, I. Stamenkovic, M. Melnick, C.B. Underhill, B. Seed, CD44 is the principal cell surface receptor for hyaluronate, Cell 61 (1990) 1303-1313.

[7] J.R. Fraser, T.C. Laurent, U.B. Laurent, Hyaluronan: its nature, distribution, functions and turnover, J Intern Med 242 (1997) 27-33.

[8] T.C. Laurent, J.R. Fraser, Hyaluronan, Faseb J 6 (1992) 2397-2404.

[9] B. Delpech, N. Girard, P. Bertrand, M.N. Courel, C. Chauzy, A. Delpech, Hyaluronan: fundamental principles and applications in cancer, J Intern Med 242 (1997) 41-48.

[10] D. Kothapalli, L. Zhao, E.A. Hawthorne, Y. Cheng, E. Lee, E. Pure, R.K. Assoian, Hyaluronan and CD44 antagonize mitogen-dependent cyclin D1 expression in mesenchymal cells, J Cell Biol 176 (2007) 535-544.

[11] L.Y. Bourguignon, H. Zhu, L. Shao, Y.W. Chen, CD44 interaction with c-Src kinase promotes cortactin-mediated cytoskeleton function and hyaluronic acid-dependent ovarian tumor cell migration, J Biol Chem 276 (2001) 7327-7336.

[12] L.Y. Bourguignon, P.A. Singleton, H. Zhu, F. Diedrich, Hyaluronan-mediated CD44 interaction with RhoGEF and Rho kinase promotes Grb2-associated binder-1 phosphorylation and phosphatidylinositol 3-kinase signaling leading to cytokine (macrophage-colony stimulating factor) production and breast tumor progression, $\mathrm{J}$ Biol Chem 278 (2003) 29420-29434.

[13] E.N. Harris, S.V. Kyosseva, J.A. Weigel, P.H. Weigel, Expression, processing, and glycosaminoglycan binding activity of the recombinant human $315-\mathrm{kDa}$ hyaluronic acid receptor for endocytosis (HARE), J Biol Chem 282 (2007) 2785-2797.

[14] C.A. Cuff, D. Kothapalli, I. Azonobi, S. Chun, Y. Zhang, R. Belkin, C. Yeh, A. Secreto, R.K. Assoian, D.J. Rader, E. Pure, The adhesion receptor CD44 promotes atherosclerosis by mediating inflammatory cell recruitment and vascular cell activation, J Clin Invest 108 (2001) 1031-1040.

[15] D. Naor, R.V. Sionov, D. Ish-Shalom, CD44: structure, function, and association with the malignant process, Adv Cancer Res 71 (1997) 241-319.

[16] G. Borland, J.A. Ross, K. Guy, Forms and functions of CD44, Immunology 93 (1998) 139-148.

[17] J. Lesley, R. Hyman, CD44 structure and function, Front Biosci 3 (1998) d616-630.

[18] M.H. Ben Mahdi, V. Andrieu, C. Pasquier, Focal adhesion kinase regulation by oxidative stress in different cell types, IUBMB Life 50 (2000) 291-299. 
[19] S.K. Mitra, D.A. Hanson, D.D. Schlaepfer, Focal adhesion kinase: in command and control of cell motility, Nat Rev Mol Cell Biol 6 (2005) 56-68.

[20] Y. Zhang, A.A. Thant, Y. Hiraiwa, Y. Naito, T.T. Sein, Y. Sohara, S. Matsuda, M. Hamaguchi, A role for focal adhesion kinase in hyluronan-dependent MMP-2 secretion in a human small-cell lung carcinoma cell line, QG90, Biochem Biophys Res Commun 290 (2002) 1123-1127.

[21] J.H. Campbell, G.R. Campbell, Culture techniques and their applications to studies of vascular smooth muscle, Clin Sci (Lond) 85 (1993) 501-513.

[22] A.H. Cory, T.C. Owen, J.A. Barltrop, J.G. Cory, Use of an aqueous soluble tetrazolium/formazan assay for cell growth assays in culture, Cancer Commun 3 (1991) 207-212.

[23] D.D. Schlaepfer, C.R. Hauck, D.J. Sieg, Signaling through focal adhesion kinase, Prog Biophys Mol Biol 71 (1999) 435-478.

[24] G.W. McLean, N.O. Carragher, E. Avizienyte, J. Evans, V.G. Brunton, M.C. Frame, The role of focal-adhesion kinase in cancer - a new therapeutic opportunity, Nat Rev Cancer 5 (2005) 505-515.

[25] K.N. Kornerup, C.P. Page, The role of platelets in the pathophysiology of asthma, Platelets 18 (2007) 319-328.

[26] S.C. Pitchford, Novel uses for anti-platelet agents as anti-inflammatory drugs, Br J Pharmacol 152 (2007) 987-1002.

[27] I. Koshiishi, M. Shizari, C.B. Underhill, CD44 can mediate the adhesion of platelets to hyaluronan, Blood 84 (1994) 390-396.

[28] A.L. Lazaar, S.M. Albelda, J.M. Pilewski, B. Brennan, E. Pure, R.A. Panettieri, Jr., T lymphocytes adhere to airway smooth muscle cells via integrins and CD44 and induce smooth muscle cell DNA synthesis, J Exp Med 180 (1994) 807-816.

[29] P.W. Noble, Hyaluronan and its catabolic products in tissue injury and repair, Matrix Biol 21 (2002) 25-29.

[30] C.L. Hawkins, M.J. Davies, Degradation of hyaluronic acid, poly- and monosaccharides, and model compounds by hypochlorite: evidence for radical intermediates and fragmentation, Free Radic Biol Med 24 (1998) 1396-1410.

[31] C. de la Motte, J. Nigro, A. Vasanji, H. Rho, S. Kessler, S. Bandyopadhyay, S. Danese, C. Fiocchi, R. Stern, Platelet-derived hyaluronidase 2 cleaves hyaluronan into fragments that trigger monocyte-mediated production of proinflammatory cytokines, Am J Pathol 174 (2009) 2254-2264.

[32] L.Y. Bourguignon, P.A. Singleton, F. Diedrich, R. Stern, E. Gilad, CD44 interaction with $\mathrm{Na}+\mathrm{-H}+$ exchanger (NHE1) creates acidic microenvironments leading to hyaluronidase- 2 and cathepsin $\mathrm{B}$ activation and breast tumor cell invasion, $\mathrm{J}$ Biol Chem 279 (2004) 26991-27007.

[33] H. Harada, M. Takahashi, CD44-dependent intracellular and extracellular catabolism of hyaluronic acid by hyaluronidase-1 and -2, J Biol Chem 282 (2007) 5597-5607.

[34] D. Kothapalli, J. Flowers, T. Xu, E. Pure, R.K. Assoian, Differential activation of ERK and Rac mediates the proliferative and anti-proliferative effects of hyaluronan and CD44, J Biol Chem 283 (2008) 31823-31829.

[35] C.L. Lin, Z.X. Zhang, Y.J. Xu, W. Ni, S.X. Chen, Focal adhesion kinase antisense oligodeoxynucleotides inhibit human pulmonary artery smooth muscle cells proliferation and promote human pulmonary artery smooth muscle cells apoptosis, Chin Med J (Engl) 118 (2005) 20-26.

[36] Z.M. Wu, X.H. Yuan, P.C. Jiang, Z.Q. Li, T. Wu, Antisense oligonucleodes targeting the focal adhesion kinase inhibit proliferation, induce apoptosis and cooperate with cytotoxic drugs in human glioma cells, J Neurooncol 77 (2006) 117-123. 
[37] Y. Fujita, M. Kitagawa, S. Nakamura, K. Azuma, G. Ishii, M. Higashi, H. Kishi, T. Hiwasa, K. Koda, N. Nakajima, K. Harigaya, CD44 signaling through focal adhesion kinase and its anti-apoptotic effect, FEBS Lett 528 (2002) 101-108. 